\title{
HSCARG downregulates NF- $\kappa$ B signaling by interacting with USP7 and inhibiting NEMO ubiquitination
}

\author{
$\mathrm{T} \mathrm{Li}^{1,2}, \mathrm{~J}$ Guan $^{1,2}, \mathrm{~S} \mathrm{Li} \mathrm{i}^{3}, \mathrm{X}$ Zhang $^{3}$ and X Zheng ${ }^{*, 1,2}$
}

Nuclear factor $\kappa \mathrm{B}(\mathrm{NF}-\kappa \mathrm{B})$ signaling is a central pathway that participates in a variety of key processes, including immunity, inflammation, cell growth and differentiation. The activity of NF- $\kappa \mathrm{B}$ is strictly regulated by a cluster of proteins, and modifications of these proteins either promote or suppress signal transduction at various steps. Here we demonstrated that HSCARG suppresses TNF $\alpha$-stimulated NF- $\kappa$ B signaling under physiological conditions. We elucidated the detailed mechanism through which HSCARG inhibits NF- $\kappa$ B activation. HSCARG interacts with NEMO and suppresses polyubiquitination of NEMO by interacting with the deubiquitinase USP7. HSACRG attenuates its inhibitory effect on NEMO ubiquitination in USP7 knockdown cells, and inhibition of NEMO polyubiquitination by USP7 is impaired in $H S C A R G^{-/-}$cells as well. Moreover, we demonstrated that USP7 is a negative regulator of TNF $\alpha$-stimulated NF- $\kappa$ B activity. Altogether, our data indicate that HSCARG and USP7 function in concert in inhibiting polyubiquination of NEMO, thus inhibiting NF- $\kappa$ B activity.

Cell Death and Disease (2014) 5, e1229; doi:10.1038/cddis.2014.197; published online 15 May 2014

Subject Category: Immunity

Nuclear factor $\kappa \mathrm{B}(\mathrm{NF}-\kappa \mathrm{B})$ is a critical regulator of multiple genes that control immunity, differentiation and other stressinduced responses. ${ }^{1-3}$ In resting cells, NF- $\kappa \mathrm{B}$ is retarded in the cytoplasm by interacting with the inhibitor of $\kappa \mathrm{B}\left(\mathrm{I}_{\kappa} \mathrm{B}\right)$ that blocks the nuclear localization signal of $\mathrm{NF}-\kappa \mathrm{B}$. In the canonical NF- $\kappa \mathrm{B}$ signaling pathway, stimuli such as tumor necrosis factor $\alpha(\mathrm{TNF} \alpha)$ recognition by the TNF receptor leads to activation of the inhibitor of $\mathrm{I}_{\kappa} \mathrm{B} \alpha$ kinase (IKK). IKK then phosphorylates $\mathrm{I} \kappa \mathrm{B} \alpha$, which results in $\mathrm{K} 48$-linked polyubiquitination of $\mathrm{I}_{\kappa} \mathrm{B} \alpha$ followed by proteasome-dependent degradation. ${ }^{4-6} \mathrm{NF}-\kappa \mathrm{B}$ dimers are then released and translocated into the nucleus, where they bind to specific DNA sequences and initiate transcription. ${ }^{7}$

The activity of NF- $\kappa \mathrm{B}$ is strictly modulated at multiple levels by variant proteins, and protein modification by ubiquitin has a key regulatory function. ${ }^{8,9}$ In addition to targeting $I_{\kappa} \mathrm{B} \alpha$ for degradation followed by NF- $\kappa$ B precursor processing, ubiquitin has a critical role in activating IKK in a proteasomeindependent manner. ${ }^{10-13}$ Initially, it was found that IKK can be activated by ubiquitination without proteasomal degradation in vitro. ${ }^{14}$ Soon after, a series of E3 ligases, which are now known as TNF receptor associate factors (TRAFs), were identified as key regulators of IKK by forming K63-linked ubiquitin chains on targeted proteins. ${ }^{15-17}$ In the TNF $\alpha$ activated $\mathrm{NF}-\kappa \mathrm{B}$ pathway, TRAF2/5 catalyzes K63-linked ubiquitination of receptor-interacting protein 1 (RIP1) and subsequently promotes the interaction between RIP1 and NEMO. ${ }^{18,19}$ The IKK complex is composed of three subunits:
$\mathrm{IKK} \alpha, \mathrm{IKK} \beta$ and the regulatory subunit NF-kappa-B essential modulator (NEMO, also known as IKK- $\gamma$ ). NEMO has a ubiquitin-binding domain that binds to polyubiquitin chains to form a huge complex that facilitates the interaction between $\operatorname{IKK} \beta$ and its kinase TGF- $\beta$-activated kinase $1 .{ }^{18,19}$ B-cell lymphoma/leukemia 10 and nucleotide-binding oligomerization domain-containing protein 2 (NOD2) are also reported to activate $\mathrm{NF}-\kappa \mathrm{B}$ by inducing $\mathrm{K} 63$-linked ubiquitin conjugation to NEMO. ${ }^{20,21}$

Several strategies have been used by cells to downregulate $\mathrm{NF}-\kappa \mathrm{B}$ and prevent persistent activation of NF- $\kappa \mathrm{B}$. For example, dephosphorylation of IKK $\beta$ by protein phosphatase $1,2 \mathrm{~A}$ or $2 \mathrm{C} \beta$ decreases IKK $\beta$ activity. ${ }^{22-24}$ Deubiquitinases (DUBs) have been reported to suppress NF- $\kappa$ B activation by removing ubiquitin chains. ${ }^{25-27}$ Among them, $A 20$ and cylindromatosis (CYLD) are the most studied DUBs that negatively regulate $\mathrm{NF}-\kappa \mathrm{B}$ activation. As a target gene of $\mathrm{NF}-\kappa \mathrm{B}, \mathrm{A} 20$ terminates NF- $\kappa \mathrm{B}$ signaling in a negative feedback loop. NF- $\kappa \mathrm{B}$ is persistently activated by $\mathrm{TNF} \alpha$ and interleukin 1 (IL-1) in A20-deficient cells, and mice lacking A20 die prematurely owing to severe inflammation and cachexia. ${ }^{28,29}$ CYLD is also an important negative regulator by preventing spontaneous activation of NF- $\kappa \mathrm{B}$. NF- $\kappa \mathrm{B}$ is constitutively activated in the absence of CYLD. ${ }^{30,31}$

HSCARG (also named NmrA-like family domain containing protein 1) is a newly identified NF- $\kappa$ B suppressor. In our previous study, we found that HSCARG inhibits TNF $\alpha$ - and IL-1-activated NF- $\kappa$ B by interacting with $\operatorname{IKK} \beta$ and

\footnotetext{
${ }^{1}$ State Key Lab of Protein and Plant Gene Research, Beijing, China; ${ }^{2}$ Department of Biochemistry and Molecular Biology, School of Life Sciences, Peking University, Beijing, China and ${ }^{3}$ College of Life Sciences, Wuhan University, Wuhan, China

${ }^{*}$ Corresponding author: Dr X Zheng, Department of Biochemistry and Molecular Biology, School of Life Sciences, Peking University, Yiheyuan Road No 5, Beijing, 100871, China. Tel: +86 106275 5712; Fax: +86 106276 5913; E-mail: xiaofengz@ @ku.edu.cn

Keywords: HSCARG; NF- $\kappa$ B; USP7; NEMO ubiquitination

Abbreviations: NF- $\kappa \mathrm{B}$, nuclear factor $\kappa \mathrm{B} ; \mathrm{I}_{\kappa} \mathrm{B}$, inhibitor of $\kappa \mathrm{B}$; IKK, inhibitor of $\kappa \mathrm{B}$ kinase; TNF $\alpha$, tumor necrosis factor $\alpha$; TRAF, TNF receptor associate factor; RIP1, receptor-interacting protein 1; NEMO, NF- $\kappa$ B essential modulator; Co-IP, co-immunoprecipitation; CYLD, cylindromatosis; IL-1, interleukin 1; HEK293T, human embryo kidney 293T; DUB, deubiquitinase; USP7, ubiquitin-specific protease 7

Received 23.10.13; revised 01.4.14; accepted 04.4.14; Edited by M Agostini
} 
suppressing its phosphorylation, ${ }^{32}$ but the detailed molecular mechanism remain to be elucidated. In this study, we show that HSCARG interacts with NEMO and suppresses polyubiquitination of NEMO. Further investigation demonstrate that HSCARG and ubiquitin-specific protease 7 (USP7; HAUSP) function together to inhibit NEMO polyubiquitination, and USP7 suppresses TNF $\alpha$-induced NF- $\kappa$ B activation. In summary, our study uncovers the suppressive mechanisms of HSCARG and USP7 in the TNF $\alpha$-activated NF- $\kappa$ B signaling pathway.

\section{Results}

HSCARG suppresses TNF $\alpha$-stimulated NF- $\kappa$ B signaling. Our previous study shows that HSCARG inhibits NF- $\kappa$ B activity. To further confirm the inhibition of $N F-\kappa B$ signaling by HSCARG under physiological conditions, the Cre/loxP system was used to generate $H S C A R G^{-/-}$HCT116 cell line by inserting an extra sequence into the fourth exon and adding further stop codons to disrupt the translation of HSCARG. Knockout of HSCARG was confirmed by PCR (Supplementary Figure S1a) and western blotting analyses (Figure 1a, left panel). And then the NF- $\kappa \mathrm{B}$ activity of $H S C A R G^{-1-}$ cells was analyzed. Upon TNF $\alpha$ treatment, $\mathrm{I}_{\kappa} \mathrm{B} \alpha$ degradation was faster in $H S C A R G^{-/-}$cells compared with that in wild-type HCT116 cells with normal HSCARG (Figure 1b, right panel). Knockout of HSCARG also retarded the de novo synthesis of $\mathrm{I} \kappa \mathrm{B} \alpha$ (Figure $1 \mathrm{~b}$ ). We generated a stable human embryo kidney (HEK) 293T cell line expressing HSCARG (Figure 1a, right panel) and examined both the endogenously and ectopically expressed HSCARG (Supplementary Figure $\mathrm{S} 1 \mathrm{~b}$ ). We found that $\mathrm{I} \kappa \mathrm{B} \alpha$ was accumulated more in stable HSCARG cells under resting state than that in HEK 293T cells. Consistently, a much higher level of $\mathrm{I}_{\kappa} \mathrm{B} \alpha$ was detected in stable HSCARG cells followed by $\mathrm{TNF} \alpha$ treatment (Figure $1 \mathrm{~b}$, lower panel).

We also found that knockout of HSCARG affected the subcellular translocation of NF- $\kappa \mathrm{B}$. $H S C A R G^{-/-}$cells were treated with $\mathrm{TNF} \alpha$, and then localization of p65 in the nucleus and cytoplasm was examined by western blotting analysis. The result showed that a higher amount of p65 translocated from the cytoplasm into the nucleus in $H S C A R G^{-/-}$cells when compared with that in control cells (Figure 1c). The observations that TNF $\alpha$-stimulated NF- $\kappa$ B activaty increased significantly in $\mathrm{HSCARG}^{-/-}$cells demonstrate that HSCARG is a strong inhibitor in the NF- $\kappa \mathrm{B}$ signaling pathway under physiological conditions.

HSCARG interacts with NEMO and inhibits its polyubiquitination. Identification of HSCARG as a strong inhibitor of NF- $\kappa$ B activity encouraged us to elucidate the detailed molecular mechanism. Both ectopic and endogenous co-immunoprecipitation (co-IP) analysis showed that HSCARG interacted with NEMO (Figure $2 \mathrm{a}$, left and middle panels, Supplementary Figure S3a), and TNF $\alpha$ treatment promoted this interaction (Figure 2a, right panel). Because NEMO can be modified by K63-linked ubiquitin chains, which is a key event for its interaction with upper intermediates and signal transduction, we next investigated whether HSCARG affected NEMO polyubiquitination. His-ubiquitin pull-down analysis showed that, in HEK 293T cells, endogenous NEMO was strongly detected with conjugated ubiquitin chains (Figure 2b, Supplementary Figure S3b). When HSCARG was overexpressed, polyubiquitin-conjugated NEMO decreased obviously, indicating that HSCARG strongly suppresses polyubiquitination of NEMO (Figure $2 b$ ). To further confirm the physiological function of HSCARG, we analyzed the variation of endogenous ubiquitinated NEMO after TNF $\alpha$ treatment. The results showed that TNF $\alpha$ treatment promoted NEMO ubiquitination, which was suppressed by ectopic expression of HSCARG (Figure 2c, Supplementary Figure $\mathrm{S} 3 \mathrm{~b})$. On the contrary, knockout of HSCARG obviously increased the level of endogenous NEMO ubiquitination (Figure 2d, Supplementary Figure S3b). Altogether, these results indicate that HSCARG interacts with the regulatory subunit NEMO of the IKK complex and inhibits NEMO ubiquitin modification.

HSCARG interacts with USP7 and inhibition of NEMO polyubiquitination by HSCARG relies on the deubiquitination activity of USP7. As described above, HSCARG interacted with NEMO and inhibited its polyubiquitination. Because protein polyubiquitination has a key role in activation, regulation and termination of the NF- $\kappa$ B pathway, and HSCARG is not a DUB, it is conceivable that HSCARG suppresses NF- $\kappa$ B by interacting with some DUBs that can regulate polyubiquitin conjugation of NEMO. Several DUBs, including A20, CYLD and USP7, have been reported to downregulate NF- $\kappa \mathrm{B}$. Co-IP assays were thus carried out to examine the interactions between these enzymes and HSCARG, and the results showed that HSCARG interacts weakly with A20 or CYLD but strongly interacts with USP7 (Figure 3a, Supplementary Figure S3c). Furthermore, the interaction between endogenous HSCARG and USP7 in cells treated with or without TNF $\alpha$ was investigated by co-IP assay. As expected, endogenous USP7 interacted with HSCARG, which was promoted by TNF $\alpha$ treatment (Figure 3b, Supplementary Figure S3d). Next, we examined whether knockdown of NEMO by siRNA affects the interaction between HSCARG and USP7. The result showed that knockdown of NEMO suppressed the binding between HSCARG and USP7, indicating an essential role of NEMO in regulating the interaction between HSCARG and USP7 (Figure 3c, Supplementary Figure S3e). We next investigated whether inhibition of NEMO polyubiquitination by HSCARG depended on USP7. In HEK 293T cells with depleted USP7 by shRNA, inhibition of NEMO polyubiquitination by HSCARG was attenuated obviously (Figure 3d, Supplementary Figure S3f). These results indicate that inhibition of NEMO ubiquitination by HSCARG relies on the deubiquitination activity of USP7.

USP7 interacts with NEMO and inhibits its polyubiquitination, and HSCARG is critical for the deubiquitination activity of USP7. We further examined whether USP7 interacted with NEMO and affected the level of NEMO polyubiquitination. USP7 and NEMO were co-transfected into HEK 293T cells, and co-IP experiment was carried out to analyze the interaction between these two proteins. We found that USP7 was immunoprecipitated with NEMO (Figure 4a, Supplementary Figure S3g). Moreover, an expression vector encoding Flag-NEMO was co-transfected with USP7 and HA-HSCARG into HEK293T cells or HSCARG ${ }^{-/-}$HEK293T cells, and then the interaction among these three proteins was 
a

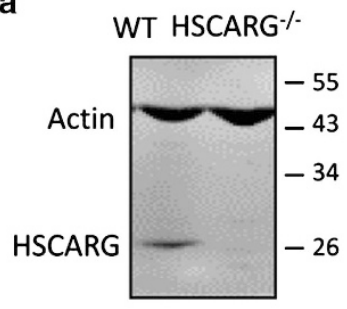

POZ-HSCARG HEK 293T

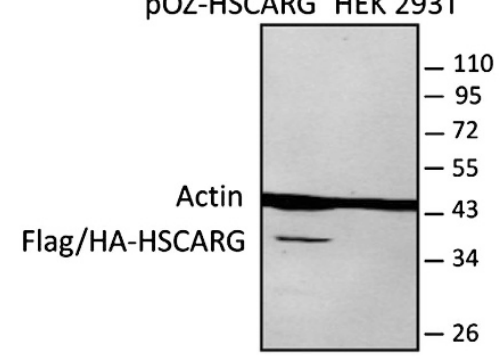

b

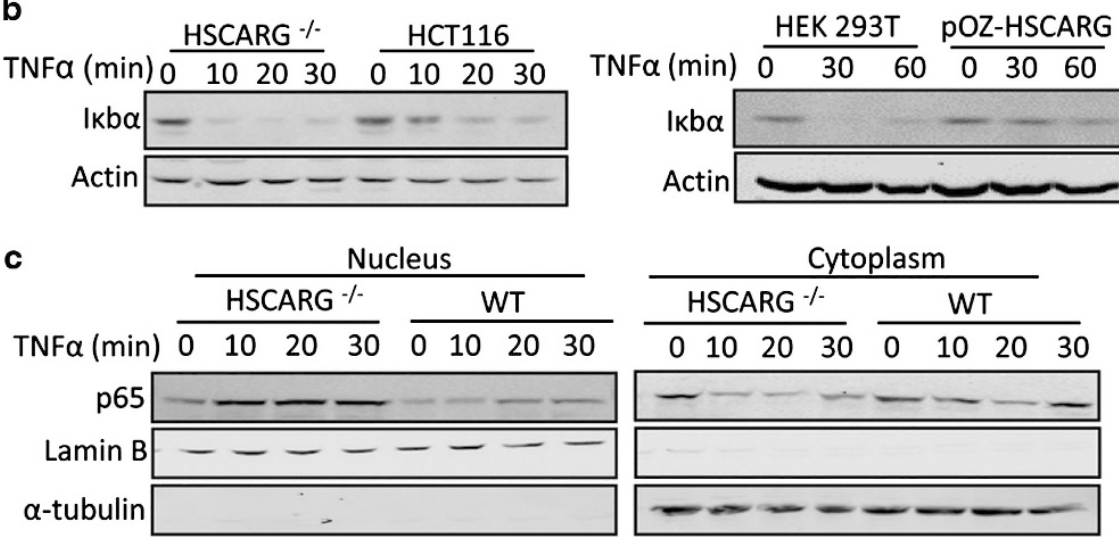

Figure 1 HSCARG is a suppressor of NF- $\kappa$ B signaling. (a) Immunoblot analysis of HSCARG expression in HSCARG ${ }^{-/-}$cells (left panel) and HSCARG-stable cells (right panel). (b) Knockout of HSCARG promotes degradation of $l_{\kappa} \mathrm{B} \alpha$ (upper panel) while stable expression of HSCARG inhibits degradation of $l_{\kappa} B \alpha$ (lower panel). Cells were treated with $\mathrm{TNF} \alpha(10 \mathrm{ng} / \mathrm{ml})$ for the indicated time, and then immunoblotting was performed using antibodies against $\mathrm{I}_{\kappa} \mathrm{B} \alpha$. Actin was used as a loading control. (c) Knockout of HSCARG results in nuclear accumulation of p65 stimulated by TNF $\alpha$. Cells were treated with TNF $\alpha(10 \mathrm{ng} / \mathrm{ml})$ for the indicated time, and then nuclear and cytoplasmic extracts were prepared. The distribution of endogenously expressed p65 was analyzed by immunoblotting using an anti-p65 antibody. Lamin B and $\alpha$-tubulin were used as nuclear and cytoplasmic controls, respectively

examined by co-IP analysis. The results showed that USP7, HSCARG and NEMO were immunoprecipitated together (Figure 4b left panel, Supplementary Figure S3h), and knockout of HSCARG impaired the binding between NEMO and USP7 (Figure 4b middle panel, Supplementary Figure S3h), indicating that these three proteins form a complex. This was confirmed by the endogenous co-IP experiments as shown in Figure 4b (right panel, Supplementary Figure S3h), in which TNF $\alpha$ treatment promoted the complex formation. To determine whether USP7 deubiquitinated NEMO polyubiquitination, we examined and compared the levels of NEMO polyubiquitination in HEK 293T cells with or without ectopic USP7. As expected, the results of ubiquitin conjugation assays showed that overexpression of USP7 strongly inhibited polyubiquitination of NEMO (Figure 4c, Supplementary Figure S3i).

The aforementioned data demonstrated that both HSCARG and USP7 inhibit NEMO polyubiquitination, and suppression of NEMO polyubiquitination by HSCARG is dependent on USP7. Next, we investigated whether HSCARG had a critical role in regulating the deubiquitination activity of USP7. The deubiquitination activity of USP7 was impaired in cells when HSCARG was knocked out (Figure 4c). Co-IP assay further confirmed that overexpression of USP7 had a weak effect on NEMO ubiquitination in $\mathrm{HSCARG}^{-/-}$ cells (Figure 4d). All these data indicate that HSCARG and USP7 function in concert in inhibiting NEMO polyubiquination, and HSCARG is important for the deubiquitination activity of USP7.

USP7 inhibits TNF $\alpha$-induced NF- $\mathrm{B}$ activation. Inhibition of NEMO polyubiquitination by USP7 suggested that USP7 probably inhibited cytokine-induced NF- $\kappa$ B activation. We hence examined the effects of USP7 on $1 \kappa \mathrm{B} \alpha$ phosphorylation, p65 cytoplasm-nuclear translocation and NF- $\kappa$ B transcriptional activity in TNF $\alpha$-treated cells (Figure 5). Compared with control cells, knockout of USP7 in HCT116 cells stimulated the degradation of $1 \kappa \mathrm{B} \alpha$, while overexpression of USP7 in 293T cells completely abolished the degradation of $\mathrm{I} \kappa \mathrm{B} \alpha$. These observations were consistent with the changes of $\mathrm{I}_{\kappa} \mathrm{B} \alpha$ phosphorylation (Figure 5a). We also found that overexpression of USP7 strongly suppressed translocation of p65 from the cytoplasm into the nucleus (Figure 5b). An NF- $\kappa \mathrm{B}$ luciferase reporter assay was carried out to analyze the effect of USP7 on NF- $\kappa$ B transcriptional activity. The result showed that USP7 suppressed TNF $\alpha$-induced NF- $\kappa$ B transcription activity in a dose-dependent manner (Figure 5c). We further confirmed the regulatory role of HSCARG on USP7 activity by examining the influence of USP7 on $1 \mathrm{kB} \alpha$ degradation in $H S C A R G^{-1-}$ cells. The results showed that knockout of HSCARG attenuated the blocking effect of USP7 and promoted the degradation of $\mathrm{IkB} \alpha$ (Figure $5 \mathrm{~d}$ ). IL-8 is a reported target of NF- $\kappa \mathrm{B} .{ }^{33}$ We also assessed the effect of USP7 on TNF $\alpha$-induced IL-8 activation, and the result showed that USP7 strongly suppressed the activation of IL-8 
a

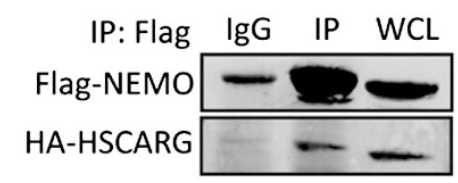

b

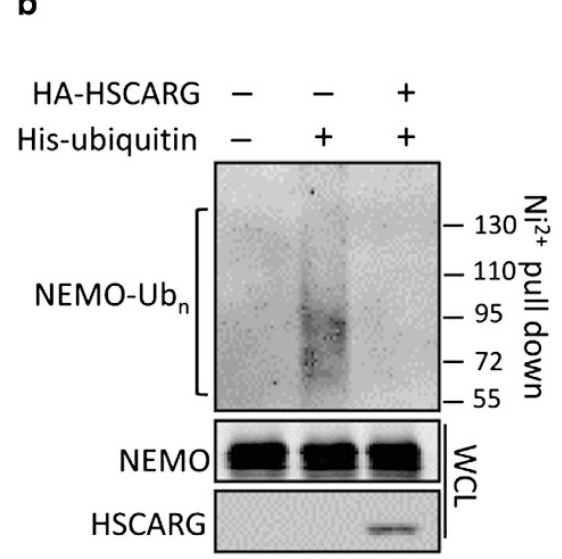

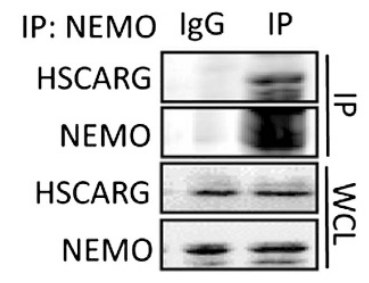

c

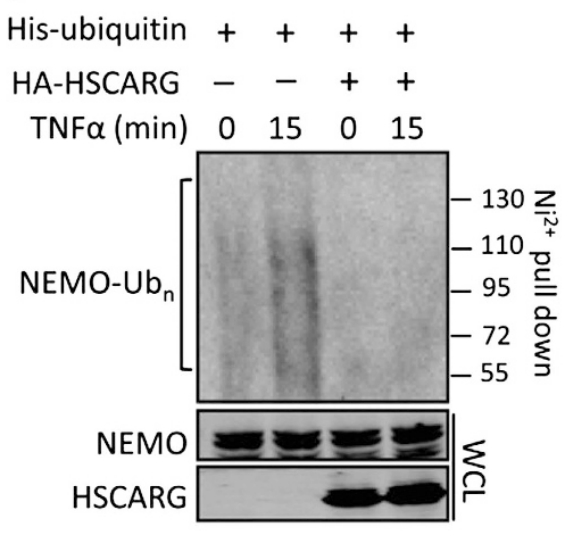

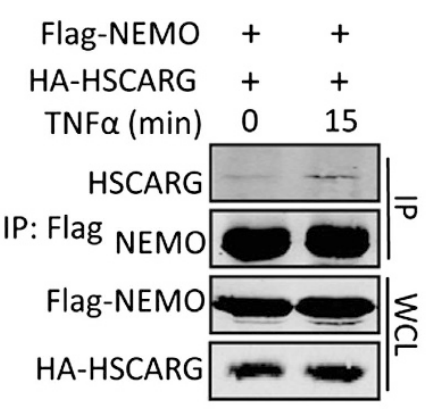

d

Figure 2 HSCARG inhibits ubiquitination of NEMO. (a) HSCARG was coimmunoprecipitated with NEMO. HEK 293T cells were transfected with the indicated plasmids. At $48 \mathrm{~h}$ after transfection, cells were lysed and immunoprecipitated with anti-Flag antibody and then analyzed by immonoblotting with the indicated antibodies (left). The endogenous interaction between HSCARG and NEMO was examined by co-IP using anti-HSCARG antibody (middle). The effect of TNF $\alpha$ treatment on the HSCARG-NEMO interaction was assessed by coIP as well (right). (b-d) HSCARG suppresses endogenous polyubiquitination of NEMO. HEK 293T cells were transfected with the indicated plasmids (b and $\mathbf{c}$ ). Endogenous ubiquitin-conjugated NEMO was enriched and analyzed by His-ubiquitin pull-down assays. Polyubiquitination of NEMO was also examined in $H_{S C A R G}{ }^{-1-}$ cells (d)

(Figure 5e). Taken together, these data indicate that USP7 inhibits TNF $\alpha$-induced NF- $\kappa \mathrm{B}$ activation and functions together with HSCARG in negatively regulating TNF $\alpha$-stimulated NF- $\kappa$ B signaling pathway.

\section{Discussion}

The NF- $\kappa \mathrm{B}$ signaling pathway responds to various stimuli, such as cytokines, and oxidative and genotoxic stresses, which results in transcription of hundreds of genes. Both activation and termination of $\mathrm{NF}-\kappa \mathrm{B}$ activity are strictly modulated at certain steps. In comparison to the many reported NF- $\kappa$ B activators, fewer suppressors have been identified. HSCARG is a newly identified NF- $\kappa$ B inhibitor. ${ }^{32}$ Here we elucidated the detailed mechanism in which HSCARG inhibits NF $-\kappa$ B activity. HSCARG interacts with USP7 and inhibits the polyubiquitination of NEMO, which decreases proteasomal degradation of $I_{\kappa} \mathrm{B} \alpha$ and further inhibits NF- $\kappa$ B activity (Figure 6). We also characterized the inhibitory function of USP7 in the TNF $\alpha$-activated NF- $\kappa$ B signal pathway.

Protein ubiquitination has important functions in positive and negative regulation of the NF- $\kappa$ B signaling pathway. ${ }^{9}$ Polyubiquitination of NEMO is essential for IKK activation that is required for activation of $\mathrm{NF}-\kappa \mathrm{B} .{ }^{21,34,35}$ In addition to K63-linked ubiquitin conjugation, NEMO is conjugated with linear ubiquitin chains by the linear ubiquitin chain assembly complex, which is also involved in TNF $\alpha$-induced NF- $\kappa \mathrm{B}$ activation. ${ }^{36,37}$ In this study, we demonstrated the suppression role of HSCARG on the ubiquitin conjugation of NEMO, indicating that HSCARG negatively regulates $\mathrm{NF}-\kappa \mathrm{B}$ signaling via inhibition of NEMO polyubiquitination. To further elucidate the detailed molecular mechanism in which HSCARG inhibits NEMO ubiquitination, we examined the interaction between HSCARG and several DUBs, including A20, CYLD and USP7, to evaluate whether HSCARG suppresses NEMO ubiquitination by interacting with some DUBs. We found that HSCARG interacts with USP7, and inhibition of NEMO polyubiquitination and NF- $\kappa$ B activity by HSCARG relies on the deubiquitination activity of USP7.

In our previous study, we demonstrated that HSCARG interacts with $\mathrm{IKK} \beta$ and suppresses its phosphorylation by promoting the interaction between PP2A and IKK $\beta,{ }^{32}$ which provided one of the possible mechanisms to explain how HSCARG inhibits IKK $\beta$ phosphorylation and NF- $\kappa$ B activity. To further investigate which protein was the direct interaction partner of HSCARG, we carried out in vitro pull-down assays between HSCARG and NEMO or IKK $\beta$. Our result showed that NEMO, but not $\operatorname{IKK} \beta$, was the direct interaction partner of HSCARG (Supplementary Figure S2a). Next, we examined whether IKK $\beta$ affected NEMO-HSCARG interaction. The result showed that knockdown of IKK $\beta$ by siRNA did not 
a

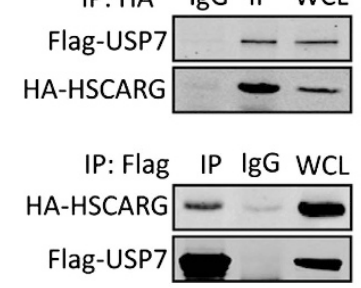

IP: Flag IgG IP WCL
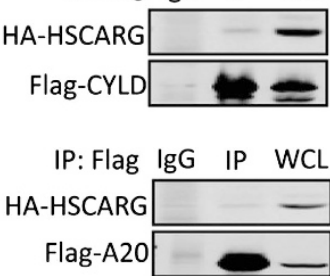

b

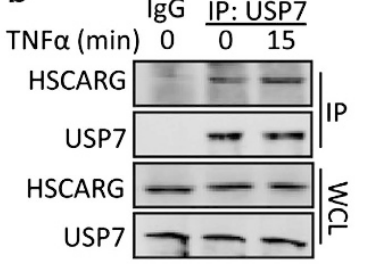

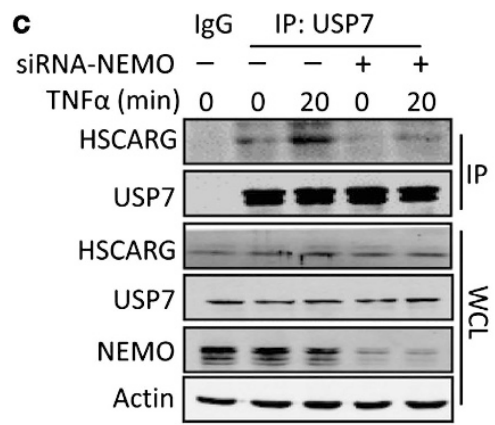

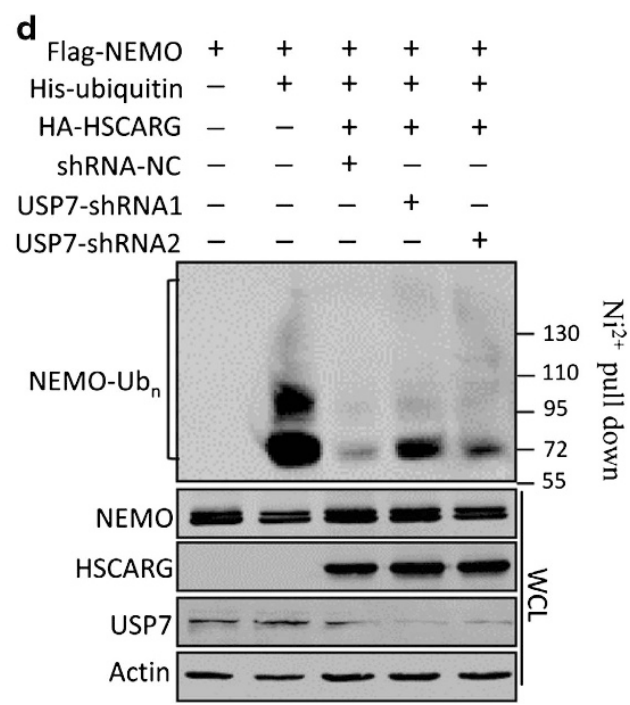

Figure 3 HSCARG interacts with USP7, and inhibition of NEMO ubiquitination by HSCARG relies on the deubiquitination activity of USP7. (a) HSCARG interacts with USP7. Co-IP assays were performed to examine the interaction between HSCARG and USP7, CYLD or A20. HEK 293T cells were transfected with the indicated expression plasmids, and then whole-cell lysates were immunoprecipitated with anti-HA or anti-Flag antibodies, followed by immunoblotting with anti-Flag or anti-HA antibodies. WCL: $5 \%$ of whole-cell lysates. (b) Interaction between endogenous HSCARG and USP7 after TNF $\alpha$ treatment. HEK293T cells were treated with or without TNF $\alpha(5 \mathrm{ng} / \mathrm{ml})$ for the indicated time, and then the endogenous interaction between HSCARG and USP7 was examined by co-IP analysis using anti-USP7 antibody. (c) NEMO is essential for the interaction of HSCARG with USP7. HEK 293T cells were transfected with control siRNA and NEMO siRNA ( $30 \mathrm{nM}$ ) for $72 \mathrm{~h}$, and then treated with or without TNF $\alpha$ ( $5 \mathrm{ng} / \mathrm{ml}$ ) for 20 min. The HSCARG-USP7 interaction was examined by co-IP using anti-USP7 antibody. (d) Inhibition of NEMO polyubiquitination by HSCARG is attenuated when USP7 is knocked down. HEK 293 T cells were transfected with the indicated plasmids, and ubiquitin-conjugated NEMO was analyzed using His-ubiquitin pull-down assay

affect the binding between HSCARG and NEMO (Supplementary Figure $\mathrm{S} 2 \mathrm{~b}$ ). These results indicate that HSCARG is likely to directly interact with NEMO and inhibit NEMO ubiquitination, which further affect IKK $\beta$ phosphorylation. With regard to the observation that HSCARG regulates $\mathrm{NF}-\kappa \mathrm{B}$ activity by affecting the degradation of RelA, ${ }^{38}$ it occurs in the nucleus, indicating a spatiotemporal regulatory effect of HSCARG on NF- $\kappa \mathrm{B}$ activation. All together, these data indicate that HSCARG regulates $\mathrm{NF}-\kappa \mathrm{B}$ pathway through different mechanisms.

USP7 has been found to participate in virus-mediated ubiquitination pathways. It has been reported that ICPO, a herpes simplex virus (HSV)-encoded protein, downregulates NEMO ubiquitination by interacting with USP7, and subsequently terminates Toll-like receptor-mediated NF- $\kappa$ B activation induced by HSV infection. ${ }^{39}$ USP7 also deubiquitinates RNA transcriptional activator-associated ubiquitin ligase and prevents it from self-ubiquitination-mediated degradation. ${ }^{40}$ Recently, USP7 was reported to regulate NF- $\kappa$ B transcription activity in the nucleus. ${ }^{41}$ Here we demonstrated the suppressive role of USP7 in the TNF $\alpha$-activated NF- $\kappa$ B signaling pathway. Our results indicate that, in addition to virus-stimulated immunity, USP7 also regulates cytokine-triggered inflammatory reactions. Our findings present an important downregulatory mechanism of $\mathrm{NF}-\kappa \mathrm{B}$ in addition to $\mathrm{A} 20$ and $\mathrm{CYLD}$.

Our study also uncovered the critical role of HSCARG in regulating USP7 activity. Inhibition of NEMO ubiquitination by HSCARG is dependent on USP7 (Figure 3c), and the deconjugation ability of USP7 is limited when HSCARG is knocked out or knocked down (Figures 4c and d). We demonstrated that HSCARG and USP7 function cooperatively in suppression of NEMO ubiquitination. These observations indicate that both HSCARG and USP7 are essential in downregulation of NEMO ubiquitination. USP7 functions as a negative regulator of IKK activation by deubiquitination, and HSCARG is a key adaptor protein that functions in a coordinate manner.

It has been reported that certain viruses inhibit NF- $\kappa$ B activity by recruitment of DUBs to escape destruction by host cells. ${ }^{39}$ Because HSCARG inhibits NF- $\kappa$ B activity by interacting with USP7, we hypothesize that HSCARG may function in virusinduced immunoreactions, which needs further investigation.

In summary, we demonstrate that HSCARG interacts with NEMO and suppresses polyubiquitination of NEMO by interacting with USP7, which further elucidate the detailed mechanism of how HSCARG inhibits NF- $\kappa$ B activity. 
b Flag-NEMO ++

pCl-USP7 ++

HA-HSCARG ++

IP: Flag IgG IP

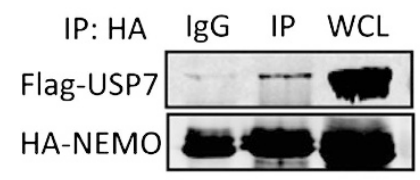

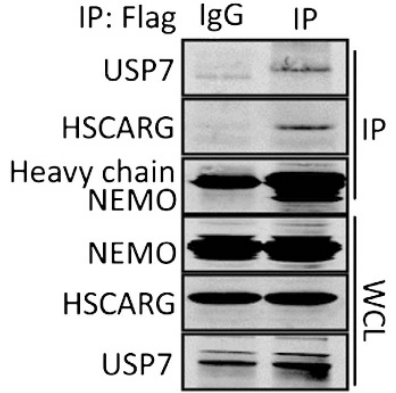

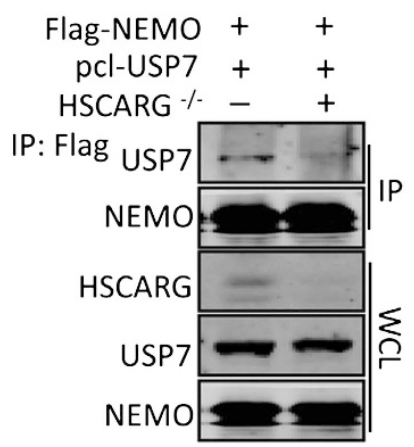

c

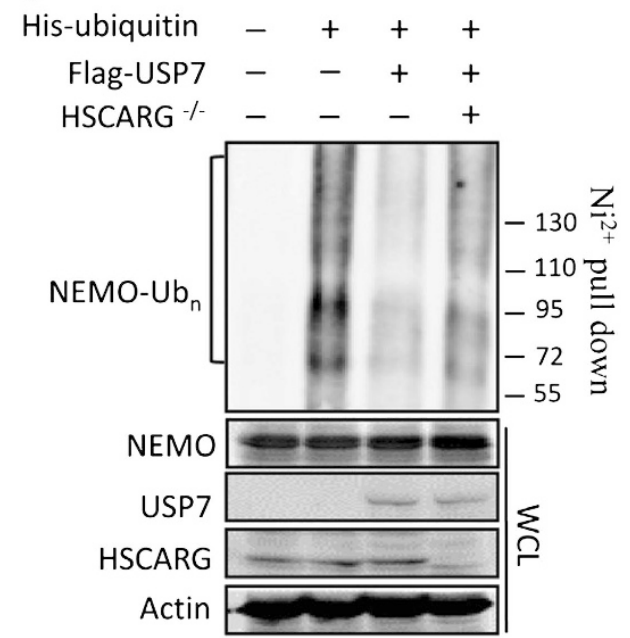

d

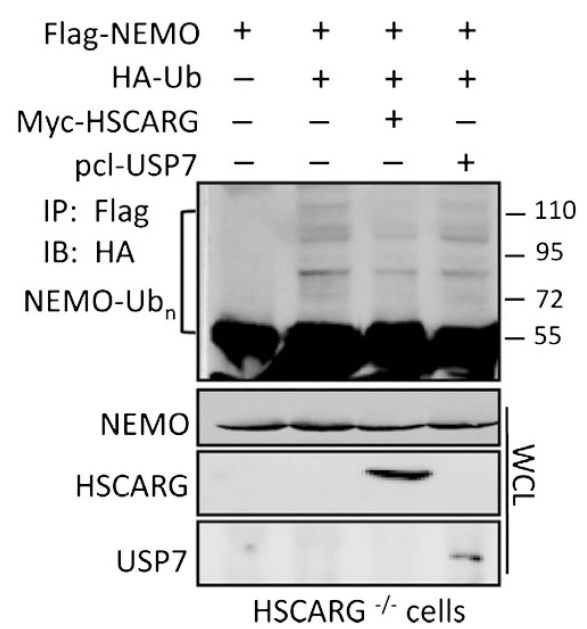

Figure 4 USP7 interacts with NEMO and inhibits NEMO polyubiquitination, and HSCARG is important for the deubiquitination activity of USP7. (a) Co-IP assay was performed to investigate the interaction between USP7 and NEMO. HEK 293T cells were transfected with the indicated plasmids. At $48 \mathrm{~h}$ after transfection, cells were harvested and lysed, and the whole supernatant was immunoprecipitated with relevant antibodies and then analyzed by immunoblotting. (b) The interaction among USP7, HSCARG and NEMO was confirmed by co-IP assay. HEK 293T cells transfected with the indicated plasmids were lysed $48 \mathrm{~h}$ after transfection, and proteins were immunoprecipitated with the indicated antibody followed by immunoblotting (left). The interaction of NEMO with USP7 was examined in HSCARG ${ }^{-1-}$ cells (middle). The effect of TNF $\alpha$ treatment on endogenous interaction among HSCARG, USP7 and NEMO was assessed by co-IP (right). (c) USP7 deubiquitinates NEMO. The wild-type and $H_{S C A R G}{ }^{-1}-$ HEK 293T cells were transfected with the indicated plasmids, and His-ubiquitin pull-down assay was performed to assess the level of NEMO ubiquitination.

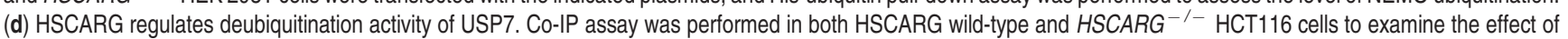
USP7 on the level of ubiquitinated NEMO

As overactivation of NF- $k$ B leads to persistent inflammation and immunoreactions that may cause damage and tumorigenesis, the important role of HSCARG in balancing NF- $\kappa$ B activation suggests that it is a potential target for treating inflammatory diseases and cancer.

\footnotetext{
Materials and Methods

Antibodies and plasmids. Mouse anti-Myc (M47-3), anti- $\alpha$-tubulin M175-3), anti-His (D291-3) and rabbit anti- $\beta$-actin were purchased from MBL (Nagoya, Japan); mouse anti-Flag (F3165) and anti-HA (H9658) from Sigma (St. Louis, MO, USA); and rabbit anti-I $\kappa \mathrm{B} \alpha$ (No.9242), anti-p-I $\kappa \mathrm{B} \alpha$ (No.2859), anti-p65 (No.4764), anti-USP7 (No.4833) and anti-NEMO (No.2685) from Cell Signaling Technology (Beverly, MA, USA). Recombinant TNF $\alpha$ and IL-1 were from PeproTech (Rocky Hill, NJ, USA). HSCARG was cloned into expression vectors pRK-HA, pRK-Flag and $\mathrm{pcDNA-Myc-His,} \mathrm{respectively,} \mathrm{and} \mathrm{verified} \mathrm{by} \mathrm{DNA} \mathrm{sequencing.} \mathrm{NEMO} \mathrm{were}$ cloned into pRK-HA or pRK-Flag expression vector. The shRNA plasmids were constructed by Shanghai Genechem Corporation (Shanghai, China). siRNA of NEMO (sc-29363) and IKK $\beta$ (sc-35644) were from Santa Cruz (Dallas, TX, USA). The sequences of the sense strand of USP7 shRNA was $5^{\prime}$-ACCCUUGGA CAAUAUUCCU-3' ${ }^{\prime}$ and $5^{\prime}$-AGUCGUUCAGUCGUCGUAU-3'.
}

Cell culture and plasmid transfection. HEK 293T and HCT116 cells were cultured in Iscove's Modified Dulbecco's Medium (Invitrogen, Carlsbad, CA, USA) with $10 \%$ fetal calf serum (HyClone, Logan, UT, USA) at $37^{\circ} \mathrm{C}$ in $5 \% \mathrm{CO}_{2}$. Plasmids were transfected using MegaTran 1.0 transfection reagent following the manufacturer's instructions (Origene, Rockville, MD, USA).

Construction of HSCARG stable HEK 293T cell line. Recombinant retroviruses expressing Flag-HA-tagged HSCARG and interleukin-2 receptor (IL-2R) $\alpha$ was constructed and transduced into HEK 293T cells. The positive cells expressing ectopic HSCARG were sorted by anti-IL-2R monoclonal antibody (13-0259, eBioscience, San Diego, CA, USA) conjugated with magnetic beads (MACS), and the expression of HSCARG were further confirmed by western blotting using anti-Flag/HA antibodies.

Co-IP assay. Cells were harvested and lysed in RIPA buffer $(50 \mathrm{mM}$ Tris-HCl, $150 \mathrm{mM} \mathrm{NaCl}, 1 \mathrm{mM}$ EDTA, $1 \%$ NP-40, pH 7.4, $1 \mu \mathrm{g} / \mathrm{ml}$ aprotinin, $1 \mathrm{mM}$ phenylmethylsulfonyl fluoride, $1 \mu \mathrm{g} / \mathrm{ml}$ leupeptin and $1 \mu \mathrm{g} / \mathrm{ml}$ pepstatin). The whole-cell lysate was precleaned by incubating with $70 \mu \mathrm{l}$ protein $\mathrm{G}$ sepharose beads (GE Healthcare, Milwaukee, MI, USA) at $4{ }^{\circ} \mathrm{C}$ for $1 \mathrm{~h}$. Antibodies $(5 \mu \mathrm{g})$ or control IgG were added to $1 \mathrm{ml}$ precleaned cell lysate for each reaction, and the sample was rotated overnight at $4^{\circ} \mathrm{C}$. Then, protein $\mathrm{G}$ sepharose beads were added and incubated for another $3 \mathrm{~h}$. The sepharose beads were pelleted by 
a

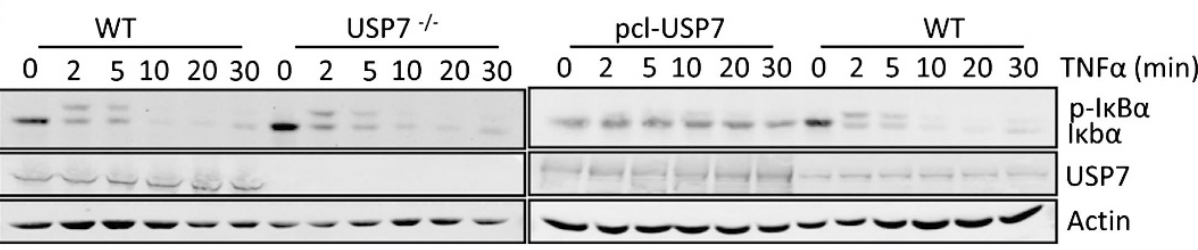

b

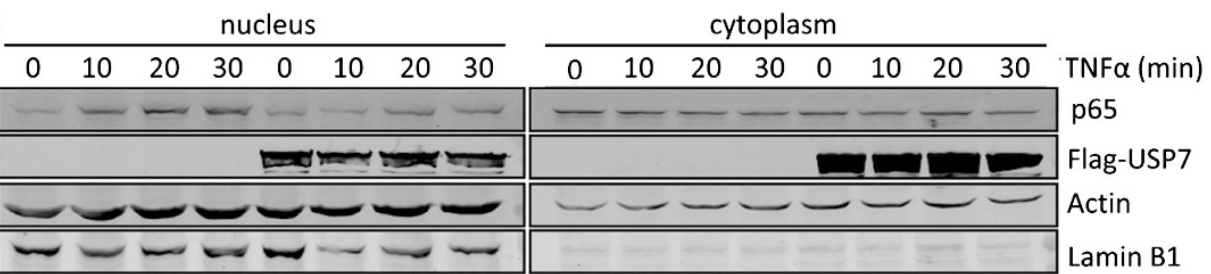

C

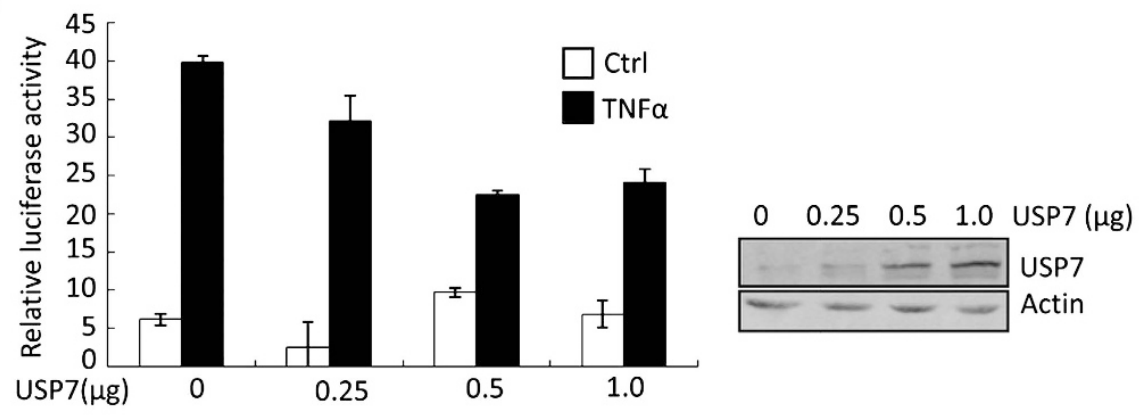

d

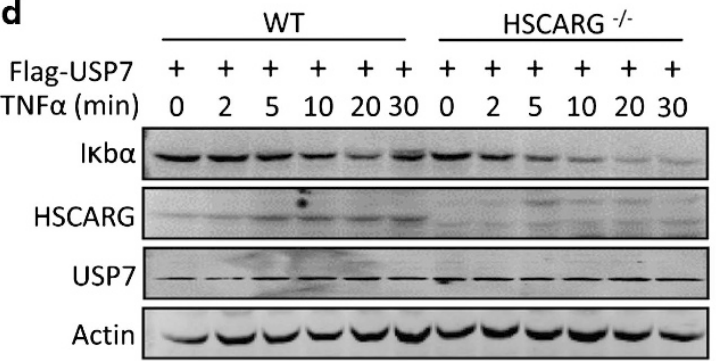

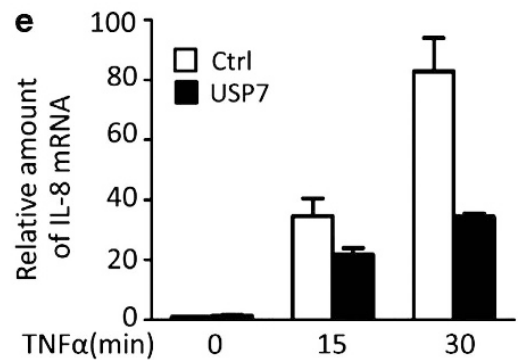

Figure 5 USP7 inhibits TNF $\alpha$-induced NF- $\kappa$ B activation. (a) USP7 suppresses phosphorylation and degradation of $\mid \kappa B \alpha$. Wild-type and USP7 ${ }^{-/-}$HCT116 cells were treated with TNF $\alpha$ (10 ng/ml) (left). 293T cells were transfected with the Flag-USP7 plasmid or empty vector and then stimulated by TNF $\alpha(10 \mathrm{ng} / \mathrm{ml})$ for the indicated times (right). Then, cells were lysed and analyzed directly by immunoblotting using anti-p- $\mid \kappa \mathrm{B} \alpha$ and anti-I $\mathrm{B} \alpha$ antibodies. Actin was used as a loading control. (b) USP7 suppresses translocation of $\mathrm{p} 65$ from cytoplasm to nucleus. HEK 293T cells were transfected with Flag-USP7 or empty plasmids, and then nuclear and cytoplasmic extracts were prepared and analyzed. (c) USP7 inhibits the TNF $\alpha$-induced NF- $\kappa$ B activation. HEK 293T cells were co-transfected with various amounts of USP7 plasmid and an NF- $\kappa B$ luciferase reporter plasmid. At $16 \mathrm{~h}$ posttransfection, cells were stimulated with or without TNF $\alpha$ for $6 \mathrm{~h}$, and then reporter activity was measured (left). Western blotting was performed to confirm the expression of USP7 (right). (d) Knockout of HSCARG impairs the blocking effect of USP7 on $1 \kappa \mathrm{B} \alpha$ degradation. HSCARG ${ }^{-/-}$cells and HEK 293T cells transfected with FlagUSP7 were treated with TNF $\alpha$ for different time and harvested at $48 \mathrm{~h}$ after transfection. The amounts of $I_{\kappa} \mathrm{B} \alpha$ were examined by immunoblot assay. (e) USP7 suppresses IL-8 transcription. HeLa cells were transfected with empty vector or Flag-USP7 and treated with or without TNF $\alpha$ for $48 \mathrm{~h}$. The mRNA of IL-8 was evaluated by RT-qPCR analysis

centrifugation at $800 \times g$ for 5 min and then washed three times with RIPA buffer. Precipitated proteins were fractionated by $12 \%$ SDS-PAGE and then transferred onto a nitrocellulose membrane (GE Healthcare). Membranes were probed with the indicated primary antibody for $2 \mathrm{~h}$ at room temperature, followed by the secondary antibody, and then detected using an Odyssey Infrared Imaging System and the software Odyssey V3.0 (LI-COR Biosciences, Lincoln, NE, USA).

His-ubiquitin pull-down assay. Cells were first transfected with a His-ubiquitin plasmid and other plasmids as indicated. At $48 \mathrm{~h}$ posttransfection, cells were harvested and washed once with phosphate-buffered saline. For each reaction $\left(1 \times 10^{6}\right.$ cells), $6 \mathrm{ml}$ cell lysis buffer $(6 \mathrm{M}$ guanidinium chloride, $0.1 \mathrm{M}$ $\mathrm{Na2HPO4/} \mathrm{NaH2PO4,} 10 \mathrm{mM}$ Tris-HCl, pH 8.0) was added, followed by incubation for $30 \mathrm{~min}$ at room temperature. $\mathrm{Ni2}+-$ nitrilotriacetate-agarose beads $(75 \mu \mathrm{l})$ were added and incubated for another $4 \mathrm{~h}$. The beads were collected by centrifugation at $2000 \times g$ for 5 min and then sequentially washed with cell lysis buffers, buffer A (8 M urea, 0.1 M Na2HPO4/ NaH2PO4, $10 \mathrm{mM}$ Tris- $\mathrm{HCl}$, pH 8.0), buffer B (8 M urea, 0.1 M Na2HPO4/ NaH2PO4, $10 \mathrm{mM}$ Tris-HCl, $\mathrm{pH} 6.3$ ) and buffer $\mathrm{C}(8 \mathrm{M}$ urea, $0.1 \mathrm{M}$ Na2HPO4/ NaH2PO4, $10 \mathrm{mM}$ Tris- $\mathrm{HCl}, 0.1 \%$ Triton $\mathrm{X}-100, \mathrm{pH} 8.0$ ). Finally, the beads were mixed with $50 \mu$ l elution buffer containing $0.2 \mathrm{M}$ imidazole, $5 \%$ SDS, $0.15 \mathrm{M}$ Tris- $\mathrm{HCl}, 10 \%$ glycerol, $\mathrm{pH} 6.7$ and boiled in SDS loading buffer. Fifteen microliters of each sample was loaded onto a $12 \%$ SDS-PAGE gel and analyzed by immunoblotting.

Luciferase reporter assay. HEK 293T cells were cultured in 24-well plates at $2 \times 10^{4}$ cells per well. After $24 \mathrm{~h}$, cells were transfected with a $1-\mu \mathrm{g}$ plasmid mixture. pRL-CMV $(5 \mathrm{ng})$ was cotransfected as a control. After $16 \mathrm{~h}$, cells were treated with $T N F \alpha(10 \mathrm{ng} / \mathrm{ml})$ for $6 \mathrm{~h}$, and then luciferase activity was assessed with a dual luciferase reporter assay kit according to the manufacturer's instructions (Promega, Madison, WI, USA). Each assay was performed in triplicate for at least three independent experiments. 


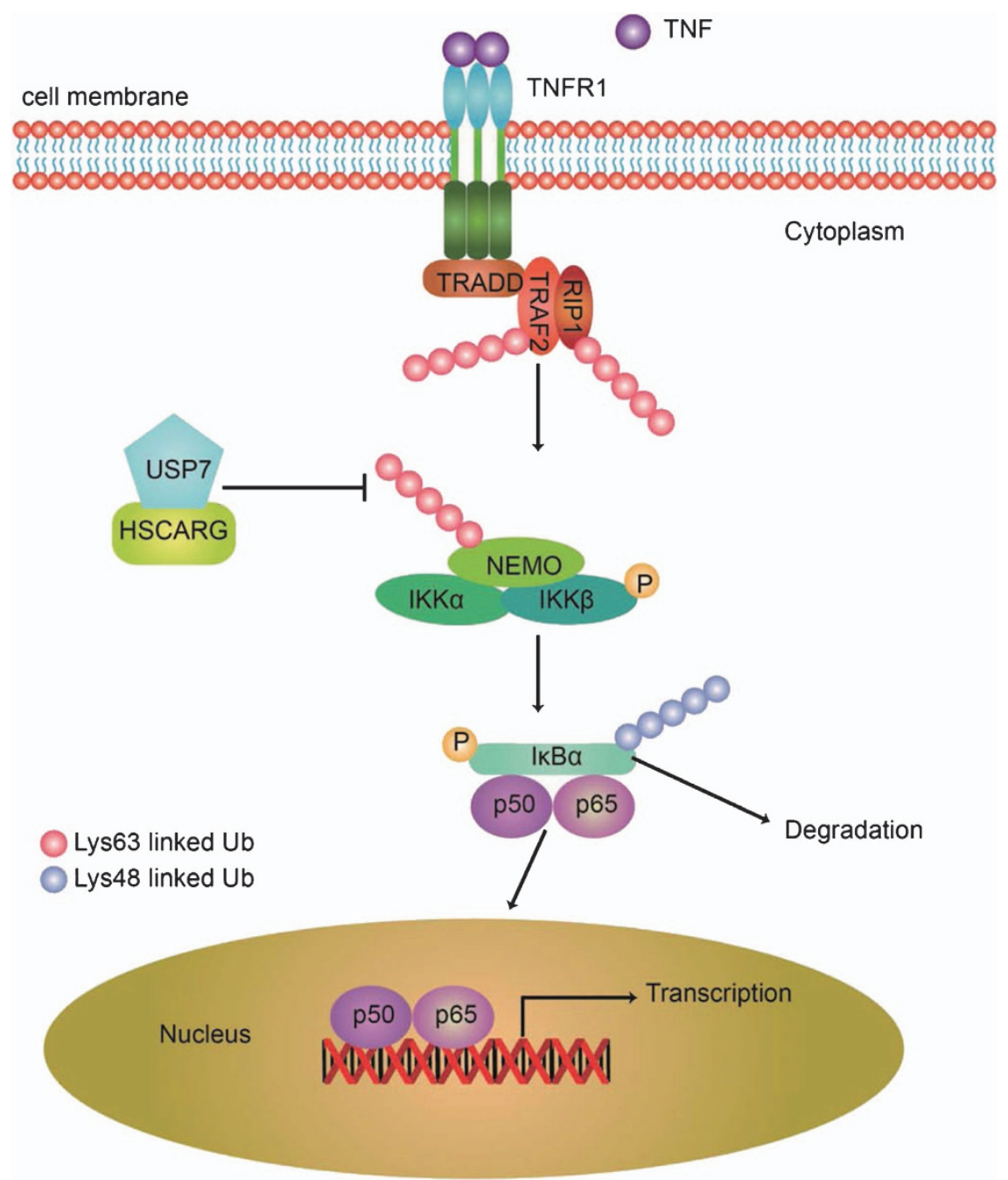

Figure 6 Model of HSCARG and USP7 in regulation of TNF $\alpha$-induced NF-אB activation. Both HSCARG and USP7 interact with NEMO and inhibit polyubiquitination of NEMO. Reduced NEMO polyubiquitination decreases proteasomal degradation of $I_{\kappa} \mathrm{B} \alpha$, which retains NF- $\kappa \mathrm{B}$ in the cytoplasm and further suppresses NF- $\kappa \mathrm{B}$ activity

Nuclear and cytoplasmic extract assay. HEK $293 \mathrm{~T}$ or HCT116 cells were cultured in $6-\mathrm{cm}$ dishes and harvested at $48 \mathrm{~h}$. Nuclear and cytoplasmic extracts were prepared using Nuclear and Cytoplasmic Extraction Reagents (Thermo Scientific, Waltham, MA, USA). Extracts were then fractioned by SDSPAGE and analyzed by immunoblotting. LaminB and $\alpha$-tubulin were used as nuclear and cytoplasmic loading controls, respectively.

Quantitative real-time PCR. RNAs were extracted using TRIzon reagent (CWBIO, Beijing, China) according to the manufacturer's instructions. The cDNAs were synthesized with FastQuant RT Kit (TIANGEN, Beijing, China), and qPCR were performed in Real-Time PCR Detection System (Bio-Rad, Hercules, CA, USA) using the SYBR Green Supermix (Bio-Rad). The primers used are as follows:

GAPDH forward primer: $5^{\prime}$-AACGGATTTGGTCGTATTGGG-3';

GAPDH reverse primer: $5^{\prime}$-TCGCTCCTGGAAGATGGTGAT-3';

IL-8 forward primer: $5^{\prime}$-CGGAAGGAACCATCTCACTGTG-3'; and

IL-8 reverse primer: 5'-AGAAATCAGGAAGGCTGCCAAG-3'.

\section{Conflict of Interest}

The authors declare no conflict of interest.

Acknowledgements. We sincerely thank Dr. Bert Vogelstein at Ludwig Center at Johns Hopkins for providing us USP7 knockout cell line. We thank
Dr. Goedele Maertens at Cancer Research UK, Dr. Danying Chen at Peking University and Dr. Zhijian Chen at University of Texas Southwestern Medical Center for providing plasmids USP7 and NEMO and ubiquitin. This work was supported by grants from the National Science Foundation of China 30930020, the National High Technology and Development Program of China 973 programs (No. 2010CB911800), Doctoral Fund of Ministry of Education of China (20130001130003) and the International Centre for Genetic Engineering and Biotechnology (ICGEB) (Project No.CRP/CHN09-01).

1. Hayden MS, Ghosh S. Shared principles in NF-kappaB signaling. Cell2008; 132: 344-362.

2. Bonizzi G, Karin M. The two NF-kappaB activation pathways and their role in innate and adaptive immunity. Trends Immunol 2004; 25: 280-288.

3. Ghosh S, May MJ, Kopp EB. NF-kappa B and Rel proteins: evolutionarily conserved mediators of immune responses. Annu Rev Immunol 1998; 16: 225-260.

4. Sun SC, Ganchi PA, Ballard DW, Greene WC. NF-kappa B controls expression of inhibitor I kappa B alpha: evidence for an inducible autoregulatory pathway. Science 1993; 259: 1912-1915.

5. Chen G, Goeddel DV. TNF-R1 signaling: a beautiful pathway. Science 2002; 296: 1634-1635

6. Karin M. How NF-kappaB is activated: the role of the IkappaB kinase (IKK) complex. Oncogene 1999; 18: 6867-6874.

7. Baud V, Karin M. Is NF-kappaB a good target for cancer therapy? Hopes and pitfalls. Nat Rev Drug Discov 2009; 8: 33-40.

8. Perkins ND. Integrating cell-signalling pathways with NF-kappaB and IKK function. Nat Rev Mol Cell Biol 2007; 8: 49-62. 
9. Chen ZJ. Ubiquitin signalling in the NF-kappaB pathway. Nat Cell Biol 2005; 7: 758-765.

10. Skaug B, Jiang X, Chen ZJ. The role of ubiquitin in NF-kappaB regulatory pathways. Annu Rev Biochem 2009; 78: 769-796.

11. Chen ZJ, Bhoj V, Seth RB. Ubiquitin, TAK1 and IKK: is there a connection? Cell Death Differ 2006; 13: 687-692.

12. Hacker H, Karin M. Regulation and function of IKK and IKK-related kinases. Sci STKE 2006; 2006: re13.

13. Windheim M, Stafford M, Peggie M, Cohen P. Interleukin-1 (IL-1) induces the Lys63-linked polyubiquitination of IL-1 receptor-associated kinase 1 to facilitate NEMO binding and the activation of IkappaBalpha kinase. Mol Cell Biol 2008; 28: 1783-1791.

14. Chen ZJ, Parent L, Maniatis T. Site-specific phosphorylation of IkappaBalpha by a novel ubiquitination-dependent protein kinase activity. Cell 1996; 84: 853-862.

15. Chung JY, Park YC, Ye H, Wu H. All TRAFs are not created equal: common and distinct molecular mechanisms of TRAF-mediated signal transduction. J Cell Sci 2002; 115: 679-688.

16. Devin A, Cook A, Lin Y, Rodriguez Y, Kelliher M, Liu Z. The distinct roles of TRAF2 and RIP in IKK activation by TNF-R1: TRAF2 recruits IKK to TNF-R1 while RIP mediates IKK activation. Immunity 2000; 12: 419-429.

17. Devin A, Lin Y, Yamaoka S, Li Z, Karin M, Liu Z. The alpha and beta subunits of IkappaB kinase (IKK) mediate TRAF2-dependent IKK recruitment to tumor necrosis factor (TNF) receptor 1 in response to TNF. Mol Cell Biol 2001; 21: 3986-3994.

18. Ea CK, Deng L, Xia ZP, Pineda G, Chen ZJ. Activation of IKK by TNFalpha requires site-specific ubiquitination of RIP1 and polyubiquitin binding by NEMO. Mol Cell 2006; 22 245-257.

19. Wu CJ, Conze DB, Li T, Srinivasula SM, Ashwell JD. Sensing of Lys 63-linked polyubiquitination by NEMO is a key event in NF-kappaB activation [corrected]. Nat Cell Biol 2006; 8: 398-406.

20. AbbottDW, Wilkins A, Asara JM, Cantley LC. The Crohn's disease protein, NOD2, requires RIP2 in order to induce ubiquitinylation of a novel site on NEMO. Curr Biol 2004; 14: 2217-2227.

21. Zhou H, Wertz I, O'Rourke K, Ultsch M, Seshagiri S, Eby M et al. Bcl10 activates the NF-kappaB pathway through ubiquitination of NEMO. Nature 2004; 427: 167-171.

22. Prajapati S, Verma U, Yamamoto Y, Kwak YT, Gaynor RB. Protein phosphatase 2Cbeta association with the lkappaB kinase complex is involved in regulating NF-kappaB activity. $J$ Biol Chem 2004; 279: 1739-1746.

23. Barisic S, Strozyk E, Peters N, Walczak H, Kulms D. Identification of PP2A as a crucia regulator of the NF-kappaB feedback loop: its inhibition by UVB turns NF-kappaB into a pro-apoptotic factor. Cell Death Differ 2008; 15: 1681-1690.

24. Li HY, Liu H, Wang CH, Zhang JY, Man JH, Gao YF et al. Deactivation of the kinase IKK by CUEDC2 through recruitment of the phosphatase PP1. Nat Immunol 2008: 9: 533-541.

25. Harhaj EW, Dixit VM. Deubiquitinases in the regulation of NF-kappaB signaling. Cell Res 21: $22-39$

26. Reyes-Turcu FE, Wilkinson KD. Polyubiquitin binding and disassembly by deubiquitinating enzymes. Chem Rev 2009; 109: 1495-1508.

27. Harhaj EW, Dixit VM. Regulation of NF-kappaB by deubiquitinases. Immunol Rev 246: 107-124

28. Lee EG, Boone DL, Chai S, Libby SL, Chien M, Lodolce JP et al. Failure to regulate TNF-induced NF-kappaB and cell death responses in A20-deficient mice. Science 2000; 289: 2350-2354.

29. Vereecke L, Beyaert R, van Loo G. The ubiquitin-editing enzyme A20 (TNFAIP3) is a central regulator of immunopathology. Trends Immunol 2009; 30: 383-391.
30. Wright A, Reiley WW, Chang M, Jin W, Lee AJ, Zhang M et al. Regulation of early wave of germ cell apoptosis and spermatogenesis by deubiquitinating enzyme CYLD. Dev Cell 2007; 13: 705-716.

31. Zhang J, Stirling B, Temmerman ST, Ma CA, Fuss IJ, Derry JM et al. Impaired regulation of NF-kappaB and increased susceptibility to colitis-associated tumorigenesis in CYLDdeficient mice. J Clin Invest 2006; 116: 3042-3049.

32. Gan Q, Li T, Hu B, Lian M, Zheng X. HSCARG inhibits activation of NF-kappaB by interacting with IkappaB kinase-beta. J Cell Sci 2009; 122: 4081-4088.

33. Wan F, Anderson DE, Barnitz RA, Snow A, Bidere N, Zheng L et al. Ribosomal protein S3: a $\mathrm{KH}$ domain subunit in NF-kappaB complexes that mediates selective gene regulation. Cell 2007; 131: 927-939.

34. Tang ED, Wang CY, Xiong Y, Guan KL. A role for NF-kappaB essential modifier/lkappaB kinase-gamma (NEMO/IKKgamma) ubiquitination in the activation of the IkappaB kinase complex by tumor necrosis factor-alpha. J Biol Chem 2003; 278: 37297-37305.

35. Sun L, Deng L, Ea CK, Xia ZP, Chen ZJ. The TRAF6 ubiquitin ligase and TAK1 kinase mediate IKK activation by BCL10 and MALT1 in T lymphocytes. Mol Cell 2004; 14: 289-301.

36. Tokunaga F, Sakata S, Saeki Y, Satomi Y, Kirisako T, Kamei K et al. Involvement of linear polyubiquitylation of NEMO in NF-kappaB activation. Nat Cell Biol 2009; 11 : 123-132.

37. Verhelst K, Verstrepen L, Carpentier I, Beyaert R. Linear ubiquitination in NF-kappaB signaling and inflammation: what we do understand and what we do not. Biochem Pharmacol 2011; 82: 1057-1065.

38. Lian M, Zheng X. HSCARG regulates NF-kappaB activation by promoting the ubiquitination of RelA or COMMD1. J Biol Chem 2009; 284: 17998-18006.

39. Daubeuf S, Singh D, Tan Y, Liu H, Federoff HJ, Bowers WJ et al. HSV ICPO recruits USP7 to modulate TLR-mediated innate response. Blood 2009; 113: 3264-3275.

40. Yu Y, Hayward GS. The ubiquitin E3 ligase RAUL negatively regulates type interferon through ubiquitination of the transcription factors IRF7 and IRF3. Immunity 2010; 33: 863-877.

41. Colleran A, Collins PE, O'Carroll C, Ahmed A, Mao X, McManus B et al. Deubiquitination of NF-kappaB by ubiquitin-specific protease-7 promotes transcription. Proc Natl Acad Sci USA 2013; 110: 618-623.

c) (i) $\Theta$ Cell Death and Disease is an open-access journal BY NC ND published by Nature Publishing Group. This work is licensed under a Creative Commons Attribution-NonCommercialNoDerivs 3.0 Unported License. The images or other third party material in this article are included in the article's Creative Commons license, unless indicated otherwise in the credit line; if the material is not included under the Creative Commons license, users will need to obtain permission from the license holder to reproduce the material. To view a copy of this license, visit http://creativecommons.org/ licenses/by-nc-nd/3.0/ 